\title{
INFLUÊNCIA DE FATORES EXTERNOS NA GERMINAÇÃO DE SEMENTES: UMA REVISÃO
}

\author{
Manoel Victor Borges Pedrosa ${ }^{1}$ \\ Melissa Alvarenga de Oliveira ${ }^{2}$ \\ Alice de Freitas Braga ${ }^{3}$ \\ Nathália Bragança Aparecida Fávaris ${ }^{4}$ \\ Lucimara Ribeiro Venial ${ }^{5}$ \\ Allan Rocha de Freitas ${ }^{6}$ \\ José Carlos Lopes ${ }^{7}$ \\ Rodrigo Sobreira Alexandre ${ }^{8}$
}

Resumo: A germinação pode ser entendida como a retomada do crescimento inicial da semente, de modo que, possua a capacidade de emitir plântulas normais em condições favoráveis. Para a realização deste estudo, tomou-se como base uma pesquisa bibliográfica onde foi analisado periódicos atuais, com o conceito Qualis da Coordenação de Aperfeiçoamento de Pessoal de Nível Superior (Capes). As sementes são afetadas negativamente por condições climáticas inadequadas, ocasionando baixa germinação e crescimento inicial limitado. O conhecimento da estrutura morfológica e do comportamento fisiológico das sementes subsidiam informações necessárias para adaptar o meio às condições necessárias para cada espécie durante o processo de germinação e crescimento.

Palavras-chave: Condições climáticas; Desenvolvimento inicial; Plântulas.

\footnotetext{
1 Biólogo, Mestrando em Produção Vegetal, Universidade Federal do Espírito Santo - UFES, Brasil. E-mail: mvborgespedrosa@gmail.com.

2 Graduanda de Engenharia Agronômica, Universidade Federal do Espirito Santo - UFES, Alegre-ES. E-mail: melissalvarengao@gmail.com.

${ }^{3}$ Graduanda de Engenharia Agronômica, Universidade Federal do Espirito Santo - UFES, Alegre-ES. E-mail: alicefreitasbraga@hotmail.com.

${ }^{4}$ Graduanda de Engenharia Agronômica, Universidade Federal do Espirito Santo - UFES, Alegre-ES. E-mail: nath-braganca@hotmail.com.

${ }^{5}$ Engenheira Agrônoma, Mestranda em Produção Vegetal, Universidade Federal do Espírito Santo - UFES, Brasil. E-mail: luci_venial@hotmail.com.

${ }^{6}$ Engenheiro Agrônomo, Dr., em Produção Vegetal, Universidade Federal do Espírito Santo - UFES, Brasil. Email: allanrochaf@gmail.com.

${ }^{7}$ Engenheiro Agrônomo, Dr., Professor do Departamento de Produção Vegetal, Centro de Ciências Agrárias, Universidade Federal do Espírito Santo - UFES, Brasil. E-mail: jcufes@bol.com.br.

${ }^{8}$ Engenheiro Agrônomo, Dr., Professor do Departamento de Ciências Florestais e da Madeira, Centro de Ciências Agrárias, Universidade Federal do Espírito Santo - UFES, Brasil. E-mail: rodrigosobreiraalexandre@gmail.com.
} 